\title{
Indicators of Health-Related Quality of Life in Cats With Degenerative Joint Disease: Systematic Review and Proposal of a Conceptual Framework
}

\author{
Gillian Yeowell ${ }^{1}$, Danielle Burns ${ }^{1}$, Francis Fatoye ${ }^{1}$, Tadesse Gebrye ${ }^{1}$, Andrea Wright ${ }^{2 *}$, \\ Kennedy Mwacalimba ${ }^{3}$ and Isaac Odeyemi ${ }^{2}$ \\ ${ }^{1}$ Department of Health Professions, Manchester Metropolitan University, Manchester, United Kingdom, ${ }^{2}$ Outcomes \\ Research, International Center of Excellence, Zoetis, Dublin, Ireland, ${ }^{3}$ Outcomes Research, US Operations, Zoetis, Dublin, \\ Ireland
}

OPEN ACCESS

Edited by:

Laura Ann Boyle,

Teagasc Food Research

Center, Ireland

Reviewed by:

Susan Hazel,

University of Adelaide, Australia

Alan J. Tilbrook,

The University of

Queensland, Australia

*Correspondence:

Andrea Wright

andrea.wright@zoetis.com

Specialty section:

This article was submitted to Animal Behavior and Welfare,

a section of the journal

Frontiers in Veterinary Science

Received: 10 July 2020 Accepted: 19 October 2021 Published: 18 November 2021

Citation:

Yeowell $G$, Burns D, Fatoye $F$ Gebrye T, Wright A, Mwacalimba K and Odeyemi I (2021) Indicators of Health-Related Quality of Life in Cats

With Degenerative Joint Disease: Systematic Review and Proposal of a

Conceptual Framework

Front. Vet. Sci. 8:582148.

doi: 10.3389/fvets.2021.582148
Objectives: The assessment of health-related quality of life (HRQOL) is becoming increasingly important in companion animals. This study describes a systematic review and development of a proposed conceptual framework to assess HRQoL in cats with osteoarthritis (OA).

Methods: The conceptual framework was developed according to published guidelines. A comprehensive search of the CAB Direct, Scopus, PubMed, and Web of Science databases was carried out for publications in English from inception to November 12, 2019. Search words used were "cat", "feline", "chronic pain", "pain", and "quality of life". Publications were selected if they were full-text and peer-reviewed, based on primary data, and identified or measured behavioral symptoms of chronic musculoskeletal pain in cats. A systematic review was conducted according to Preferred Reporting Items for Systematic Reviews and Meta-Analyses (PRISMA) guidelines. A data extraction form was developed from categories identified in the literature review and piloted on a small number of studies to ascertain the appropriateness for relevant data extraction. Categories were then finalized, and key domains were identified. The domains were then synthesized to develop a conceptual framework.

Results: A total of 454 studies were identified, of which 14 met the eligibility criteria and were included in the meta-synthesis. All 14 were assessed to be of good quality. Seven domains related to HRQoL in cats with OA were thematically identified from the data: mobility, physical appearance, energy and vitality, mood, pain expression, sociability, and physical and mental wellbeing. The three main HRQoL domains were pain expression, mobility, and physical and mental wellbeing, which impacted all the others. Pain and mobility impacted all six other domains, with increased pain and decreased mobility negatively impacting physical appearance, energy and vitality, mood, sociability, and physical and mental wellbeing. 


\begin{abstract}
Conclusions and Relevance: This is the first study to develop an evidence-based conceptual framework for the assessment of HRQoL in cats with OA. The proposed conceptual framework suggests that effective management of chronic pain in cats may improve their overall HRQoL.
\end{abstract}

Keywords: pain, health-related quality of life (HRQoL), cats, osteoarthritis (OA), conceptual framework

\section{INTRODUCTION}

The feline medicalized population is an aging population, primarily due to increasing life expectancy in companion animals as a benefit of improved animal healthcare $(1,2)$. One condition associated with advancing years in cats is degenerative joint disease (DJD) or osteoarthritis (OA) $(1,3)$. Aging cats are more likely to show joint changes consistent with $\mathrm{OA}$, and radiographic evidence of OA has been found in an estimated $90 \%$ of cats over the age of 12 (4).

$\mathrm{OA}$ is associated with chronic pain, which, in turn, can negatively impact health-related quality of life (HRQoL) (1). HRQoL is a multi-dimensional concept and includes factors such as pain and physical activity, and mental wellbeing $(2,5)$.

The assessment of HRQoL is becoming increasingly important in companion animals (2). HRQoL can be assessed using questionnaires developed with pet owners who then observe and report on the physical and emotional impacts of specific diseases like OA. However, a recent systematic review of quality of life (QoL) in cats by Doit et al. (6) highlights that within the literature, there is a lack of consensus regarding HRQoL in cats, and among the validated measures reviewed, one specific to assess OA has not been identified. Therefore, the first step in understanding the HRQoL impacts of OA is to develop a conceptual framework that proposes how chronic pain influences physical and mental wellbeing, based on a systematic literature review of QoL and OA in cats.

In general, the assessment of chronic pain in cats with OA is not straightforward as lameness is uncommon (3). Symptoms develop gradually and, as such, can be overlooked by pet owners and veterinarians (7-9). Furthermore, clinical detection relies primarily on owner reports, but subtle and non-specific symptoms may be incorrectly attributed to aging (10).

Therefore, there is need for research that identifies the factors that contribute to HRQoL in cats with OA. This will enable evaluations on how OA impacts HRQoL and, ultimately, the effective assessment of treatment interventions for cats with this condition. The aim of this study was to propose a conceptual framework for a tool that can assess HRQoL in cats with OA.

\section{MATERIALS AND METHODS}

The conceptual framework was developed according to published guidelines $(11,12)$. Development was undertaken in two phases. Phase 1 was a meta-synthesis that involved mapping the selected data sources, categorizing the data, and thematically identifying and naming key domains. Phase 2 was a synthesis of domains to develop the conceptual framework.

\section{Phase 1: Meta-Synthesis}

Mapping of data sources was accomplished first by conducting a systematic review to identify the relevant literature. The review was conducted in accordance with the principles set out for reporting systematic reviews and meta-analyses, Preferred Reporting Items for Systematic Reviews and Meta-Analyses (PRISMA) guidelines (13).

\section{Inclusion and Exclusion Criteria}

Studies were eligible for inclusion in the meta-analysis if the following criteria were met: domestic cat was the main population; full-text, peer-reviewed journal article; articles based on primary data; measured/identified behavioral symptoms of chronic musculoskeletal pain; and articles were written in English. Articles were excluded if domestic cat was not the main population; article was not a full-text peer-reviewed journal article (e.g., conference paper, book chapter, citation only, or opinion piece); articles based on secondary data, e.g., reviews; cats were anesthetized; medical/physiological investigations of chronic MSK pain; article was not concerned with chronic MSK pain (i.e., acute and postoperative pain); focused on treatment/drug efficacy on chronic MSK pain; or was not written in English.

A comprehensive search strategy was developed to ensure that all relevant sources of data were identified. Searches were undertaken from inception to November 12, 2019 (date of the search) via the following electronic databases: CAB Direct, Scopus, PubMed, and Web of Science. The reference lists of all included studies, any relevant systematic reviews, and key background papers were scrutinized to identify any additional relevant studies. The keywords used were "cat", "feline", "chronic pain", "pain", and "quality of life". These keywords were used in various combinations with Boolean operators "OR" and "AND" used to combine search terms.

\section{Study Selection}

All searches were exported into EndNote Web (Thomas Reuter, CA, USA) bibliography software. Duplicates of articles were removed electronically and manually. Study selection was carried out in two stages. The first stage included title and abstract screening. To ensure that the relevant studies were identified and selected, two researchers independently screened titles and abstracts for eligibility based on the inclusion/exclusion criteria (DB and TG). Full-text articles were obtained and independently screened against the eligibility criteria (DB and TG). Manual searching of the reference lists was undertaken to identify any additional studies. Any disagreements in study selection between the researchers were resolved through discussion and 


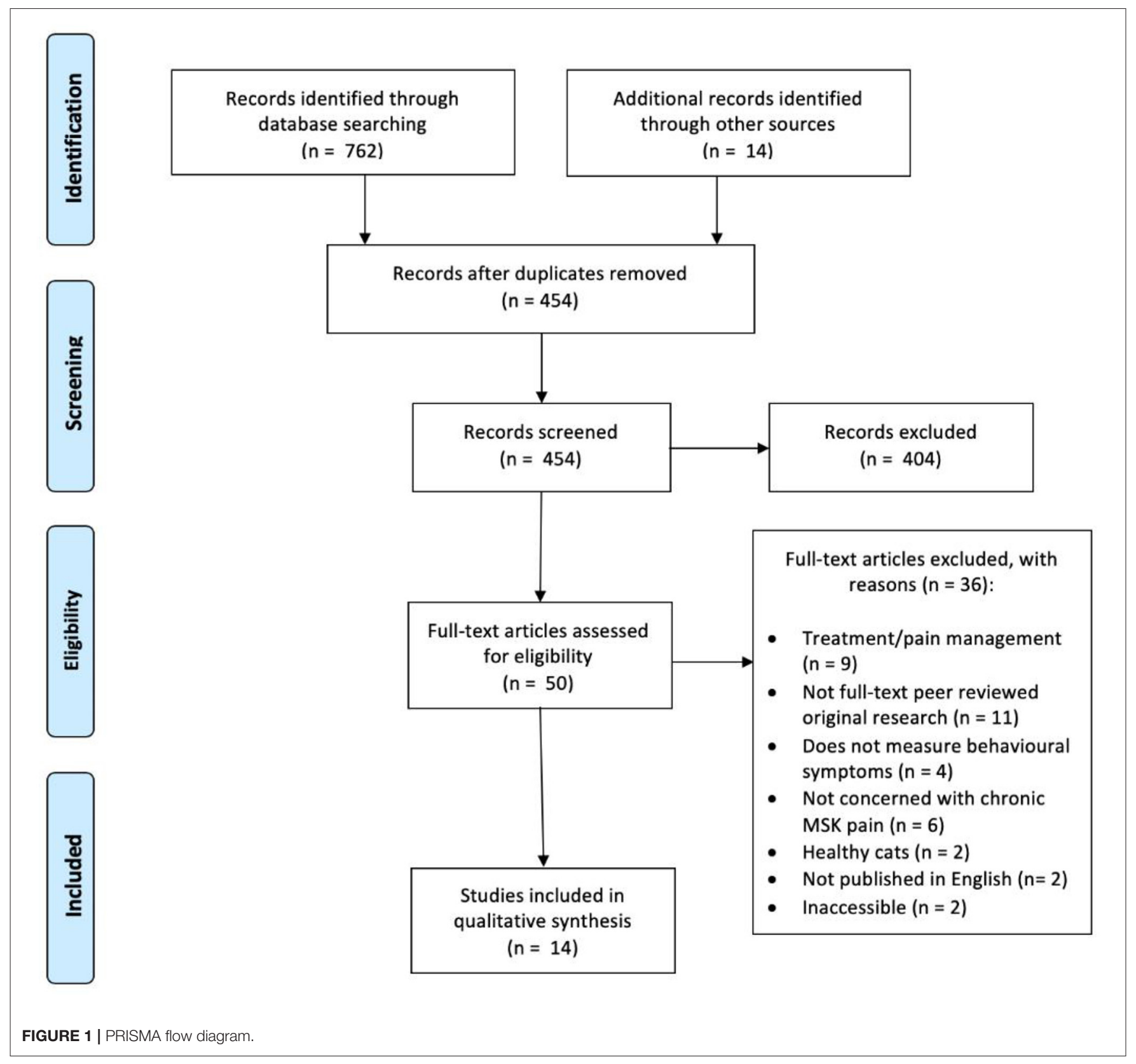

consultation with two members of the project team (GY and FF). Stages in the study selection are illustrated in Figure 1 (PRISMA flow chart) (13).

\section{Data Extraction}

A data extraction form was developed from categories identified during the review of the literature $(5,14,15)$. This was piloted on a small number of studies to ascertain the appropriateness for extracting relevant information. Piloting revealed some redundant categories (where no items were identified within them); categories that were conceptually similar and could be collapsed into one category; and the need for additional categories whereby items were not captured. The initial categories were mobility, emotion, energy, engagement, appetite, coat, eyes, fitness, sleep, and pain behavior. These were refined to mobility, physical appearance, energy and vitality, mood, pain expressions, sociability, and physical and mental wellbeing (Table 1). Data were extracted by one researcher (DB) and independently checked by a second researcher (TG). Two members of the project team (GY and FF) were available if there were disagreements, but were not required.

\section{Quality Assessment}

The methodological quality of the identified studies was critically appraised using the criteria listed in the ARRIVE guideline for 
TABLE 1 | Narrative of conceptual framework.

Health-related quality of life consisted of seven domains.

Three main domains were pain expression, mobility, and physical and mental wellbeing

Pain expression impacted on: Physical and mental wellbeing, mobility, sociability, mood, physical appearance, energy and vitality

Pain expression was impacted by: Mobility

Physical and mental wellbeing impacted on: Mood

Physical and mental wellbeing was impacted by: Pain, mobility, sociability, mood, energy and vitality

Mobility impacted on: Pain expression, physical and mental wellbeing, physical appearance, energy and vitality, sociability, mood

Mobility was impacted by: Pain, energy and vitality

Mood impacted on: Physical and mental wellbeing, energy and vitality, sociability

Mood was impacted by: Pain, mobility, physical and mental wellbeing, energy and vitality

Physical appearance impacted on: Physical and mental wellbeing

Physical appearance was impacted by: Pain, mobility, energy and vitality

Energy and vitality impacted on: Physical and mental wellbeing, mobility

Energy and vitality was impacted by: Pain, mobility, mood

Sociability impacted on: Physical and mental wellbeing

Sociability was impacted by: Pain, mobility, mood, energy and vitality

reporting animal research (16) and the veterinary (STROBEVet) statement (17). The ARRIVE guideline contains 20 criteria and can be used to evaluate studies of bioscience research using laboratory animals and comparative experiments, whereas the Veterinary (STROBE-Vet) statement can be applied for observational studies. A score of 1 was assigned if they fulfilled the requirement of reporting for the item completely, and 0 for not reporting. The maximum score for an article that reported all information was 24 for the STROBE-Vet statement and 20 for the ARRIVE guideline. A cutoff point score of $50 \%$ was used to assess the quality of the included studies (18). Studies were determined to be of "good quality" if they scored $\geq 50 \%$, whereas studies were considered "poor quality" studies if they scored $<50 \%$ (18). The final step in Phase 1 was to categorize the data and confirm the key domains.

\section{Phase 2: Synthesis of Domains to Develop Conceptual Framework}

In this phase, a thematic analysis framework, as described by Braun and Clarke (19), was used to inductively analyze the extracted data (Supplementary File 1) to identify HRQoL domains. The data were synthesized into a narrative under each domain to develop the conceptual framework (Figure 2). The approach to narrative synthesis has recently been described elsewhere (20). The proposed conceptual framework underwent preliminary review with three stakeholders.

\section{Ethics}

Ethical approval was obtained from the University Faculty Health, Psychology and Social Care Research Ethics and Governance Committee (UK): Reference Number: 10368.

\section{RESULTS}

A total of 776 studies were identified. After removing duplicates, and applying the inclusion criteria and abstract screening, 14 studies met the inclusion criteria (Figure 1) and were included in the meta-synthesis (Supplementary Material Table 2).

Of the 14 studies included, six studies were from the USA $(15,21-25), 4$ were from the UK $(1,5,26,27)$, three studies were from Canada $(10,28,29)$, and there was one study from the Netherlands (30). Eleven studies were observational and three were comparative studies. The total number of cats included was 1,168 , and the number of cat owners was 324 .

The scores of the methodological quality assessment are presented (Supplementary Materials). Eight studies (1, 10, 15, $21-24,29)$ achieved more than $70 \%$ of the checklist criteria. The remaining six studies $(5,25-28,30)$ scored between 50 and $70 \%$ of the criteria. All included studies scored $\geq 50 \%$ and, as such, met the criteria to be considered good quality (18).

\section{Categorizing the Data and Identifying and Naming Key Domains}

From the data extracted, data were categorized, and key domains were confirmed (Figure 3). Seven domains were identified from the data: mobility, physical appearance, energy and vitality, mood, pain expression, sociability, and physical and mental wellbeing. The items that formed these domains are presented in Figure 3.

\section{Synthesis of Domains to Develop Conceptual Framework}

Key domains were synthesized to develop the conceptual framework (Figure 2). The three main domains in terms of frequency and impact were pain expression, mobility, and physical and mental wellbeing. A narrative of how these domains interact is presented in Table $\mathbf{1}$.

Initial validation of the conceptual framework was undertaken. The conceptual framework was shared with three key stakeholders: a veterinarian practicing in companion animals (UK based), an owner of an aged cat (15 years) (UK based), and a representative from a pharma company specializing in animal pharma (USA based), to obtain their views and feedback. They confirmed that all domains were relevant and their relationship was appropriate. No changes were requested. All participants confirmed the conceptual framework accurately and wholly reflected the domains that impact HRQoL in cats with OA. A subsequent qualitative concept elicitation study with key informants including veterinarians and cat owners will help us confirm that the HRQoL domains and areas of overlap in our model reflect real-world experiences. Thus, our framework may be considered preliminary until we can further validate it with qualitative evidence.

\section{DISCUSSION}

To the authors' knowledge, this is the first study to develop an evidence-based conceptual framework for the assessment of HRQoL in cats with OA. To achieve this, a systematic review 


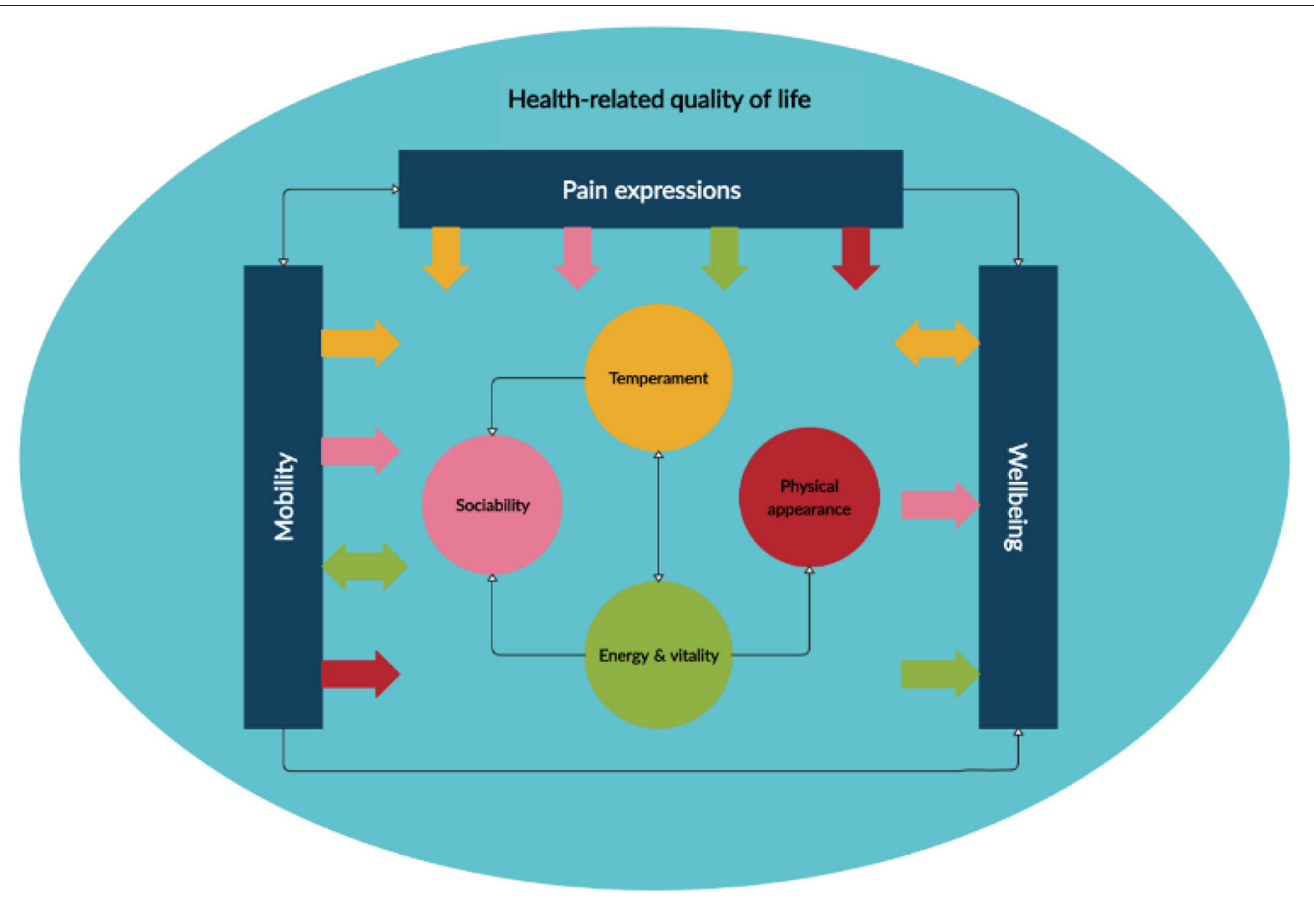

FIGURE 2 | Conceptual framework.

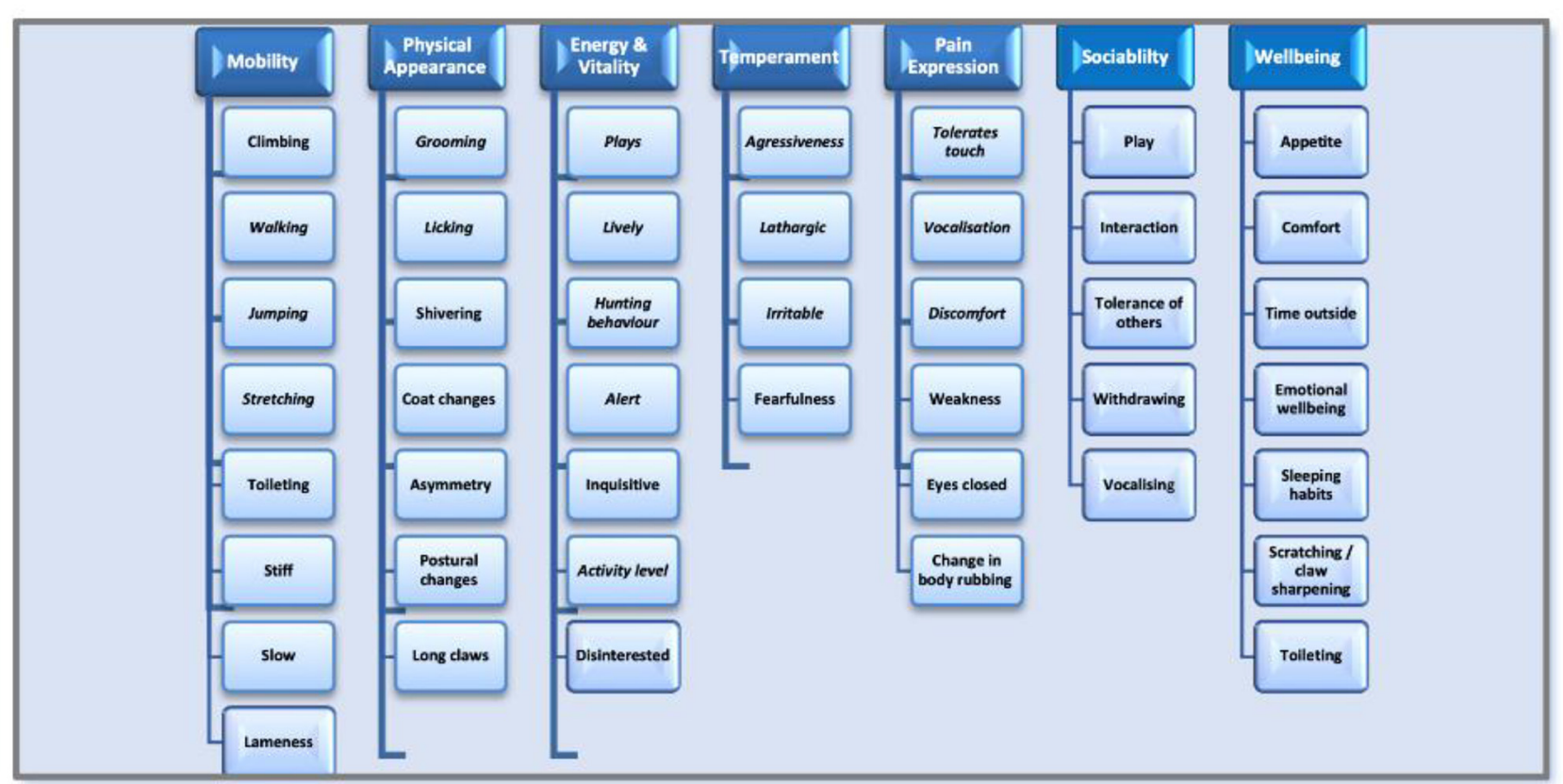

FIGURE 3 | Quality-of-life domains.

was undertaken to identify the relevant literature to develop the conceptual framework. Fourteen articles were identified, all of which were assessed as good quality. From the data extracted, seven domains were identified in relation to HRQoL in cats with
OA. These domains were mobility, physical appearance, energy and vitality, mood, pain expression, sociability, and physical and mental wellbeing. Of these, three domains, pain expression, mobility, and physical and mental wellbeing, were identified as 
being more important to HRQoL in cats with OA. Feline physical and mental wellbeing was impacted by five of the six domains. This preliminary conceptual framework was found to be a valid representation of HRQoL in cats with OA. A similar conceptual framework development approach has recently been described in human and animal health $(20,31)$.

There is evidence that the relationship between pain and QoL is complex (32). This study adds to this evidence in relation to chronic pain and HRQoL in cats. Pain and mobility were identified as impacting on all other domains, with increased pain and decreased mobility negatively impacting physical appearance, energy and vitality, mood, sociability, and physical and mental wellbeing. Pain is only one of the main domains that impacts HRQoL. Other domains can also negatively impact HRQoL. However, this conceptual framework suggests that effectively managing chronic pain in cats may improve their HRQoL. In addition, if pain is reduced, the other domains of HRQoL, for example, mobility and physical and mental wellbeing, may also improve.

There is a lack of research that has investigated HRQoL with $\mathrm{OA}$ in cats. From the systematic review, only 14 studies were identified. Of these, only one study (15) looked at HRQoL and OA. Benito et al. (15) undertook a cross-sectional study to identify items considered important for their cat's QoL by owners of cats with OA. However, the study focused on QoL in relation to mobility. In addition, $95 \%$ of the cat owners surveyed indicated that their cats were indoor cats. This limits the generalizability of this study to the wider population of cats.

The remaining studies focused on pain and DJD or OA (10, $21,22,25,29)$, pain and behavior change $(1,27)$ spondylosis and behavior change (30), clinical signs and $\mathrm{OA}(26,28)$ activity and painful OA (24) and the development of a generic instrument to measure HRQoL in a range of chronic diseases (5).

There were some limitations to this study. Only articles published in English were included; therefore, there is the possibility that some further data were available to inform the conceptual framework. However, preliminary review of the conceptual framework suggests that it was a comprehensive and accurate reflection of the domains that impact HRQoL in cats with DJD. A subsequent qualitative concept elicitation study with key informants including veterinarians and cat owners will help us confirm that the HRQoL domains and areas of overlap in our model reflect real-world experiences. Then, the development of

\section{REFERENCES}

1. Bennett D, Morton C. A study of owner observed behavioural and lifestyle changes in cats with musculoskeletal disease before and after analgesic therapy. J Feline Med Surg. (2009) 997-1004. doi: 10.1016/j.jfms.2009. 09.016

2. Tatlock S, Gober M, Williamson N, Arbuckle R. Development, and preliminary psychometric evaluation of an owner-completed measure of feline quality of life. The Veterinary J. (2017) 22-32. doi: $10.1016 /$ j.tvjl.2017.10.005

3. Lascelles B. Feline degenerative joint disease. Vet Surg. (2010) 2-13. doi: 10.1111/j.1532-950X.2009.00597.x an evidence-based tool to assess HRQoL in cats with OA can be undertaken.

\section{CONCLUSION}

This study has developed a conceptual framework for a tool that can assess HRQoL in cats with OA. Seven domains were identified in relation to HRQoL in cats with OA: mobility, physical appearance, energy and vitality, mood, pain expression, sociability, and physical and mental wellbeing. The three main domains were pain expression, mobility, and physical and mental wellbeing. This conceptual framework suggests that effective management of chronic pain in cats with OA may improve their HRQoL. However, other domains can also negatively impact HRQoL. The findings of this study can be used to inform the development of an evidence-based tool to assess HRQoL in cats with OA.

\section{DATA AVAILABILITY STATEMENT}

The original contributions presented in the study are included in the article/Supplementary Material, further inquiries can be directed to the corresponding authors.

\section{AUTHOR CONTRIBUTIONS}

GY: study design, data analysis, and manuscript development. DB and TG: data collection, data analysis, and final report preparation. FF: study design, data collection, data analysis, and manuscript development. AW: study design, quality assurance, and manuscript preparation. KM: quality assurance and manuscript preparation. IO: study design and manuscript development.

\section{FUNDING}

The authors declare that this study was funded by Zoetis.

\section{SUPPLEMENTARY MATERIAL}

The Supplementary Material for this article can be found online at: https://www.frontiersin.org/articles/10.3389/fvets. 2021.582148/full\#supplementary-material
4. Bolton S. Management of the cat with osteoarthritis. Veterinary Nursing J. (2016) 266-70. doi: 10.1080/17415349.2016.1195712

5. Noble CE, Wiseman-Orr LM, Scott ME, Nolan AM, Reid J. Development, initial validation and reliability testing of a web-based, generic feline healthrelated quality-of-life instrument. J Feline Medicine and Surgery. (2019) 84-94. doi: $10.1177 / 1098612 X 18758176$

6. Doit H, Dean RS, Duz M, Brennan ML. A systematic review of the quality of life assessment tools for cats in the published literature. Veterinary J. (2021) 272:105658. doi: 10.1016/j.tvjl.2021.105658

7. Epstein M, Rodan I, Griffenhagen G, Kadrlik J, Petty M, Robertson S, et al. 2015 AAHA/AAFP Pain management guidelines for dogs and cats J Feline Med Surg. (2015) 251-72. doi: 10.1177/1098612X15572062 
8. Merola I, Mills DS. Systematic review of the behavioural assessment of pain in cats. J Feline Med Surg. (2015) 1-17. doi: 10.1177/1098612X15578725

9. Addison ES, Clements DN. Repeatability of quantitative sensory testing in healthy cats in a clinical setting with comparison to cats with osteoarthritis. J Feline Med Surg. (2017) 19:1274-82. doi: 10.1177/1098612X17690653

10. Klinck MP, Monteiro BP, Lussier B, Guillot M, Moreau M, Otis C, et al. Refinement of the Montreal Instrument for Cat Arthritis Testing, for Use by Veterinarians: detection of naturally occurring osteoarthritis in laboratory cats. J Feline Med Surg. (2018) 20:728-40. doi: 10.1177/1098612X17730172

11. Jabareen Y. Building a conceptual framework: Philosophy, definitions and procedure. Int J Qualitat Met. (2009) 8:49-62. doi: 10.1177/160940690900800406

12. Ravitch SM, Riggan JM. Reason \& rigor: How conceptual frameworks guide research. Los Angeles: Sage (2016).

13. Moher D, Liberati A, Tetzlaff J, Altman DG, PRISMA Group. Preferred reporting items for systematic reviews and meta-analyses: the PRISMA statement. Int J Surg. (2010) 8:336 rat doi: 10.1016/j.ijsu.2010.02.007

14. Freeman LM, Rodenberg C, Narayanan A, Olding J, Gooding MA, Koochaki P. Development E, and initial validation of the Cat HEalth and Wellbeing (CHEW) Questionnaire: a generic health-related quality of life instrument for cats. J Feline Med Surg. (2016) 689-701. doi: 10.1177/1098612X16657386

15. Benito J, Gruen ME, Thomson A, Simpson W. Lascelles BD. Ownerassessed indices of quality of life in cats and the relationship to the presence of degenerative joint disease. J Feline Med Surg. (2012) 14:863urgE doi: 10.1177/1098612X12453904

16. Kilkenny C, Browne WJ, Cuthill IC, Emerson M, Altman DG. Improving bioscience research reporting: the ARRIVE guidelines for reporting animal research. PLoS Biology. (2010) 8:e1000412. doi: 10.1371/journal.pbio.1000412

17. Sargeant JM, O'Connor AM, Dohoo IR, Erb HN, Cevallos M, Egger M, et al. Methods and processes of developing the Strengthening the Reporting of Observational Studies in Epidemiology-Veterinary (STROBE-Vet) statement. Journal Vet Intern Med. (2016) 30:1887-95. doi: 10.1111/jvim.14574

18. Horn J, De Haan RJ, Vermeulen M, Luiten PG. Limburg M. Nimodipine in animal model experiments of focal cerebral ischemia: a systematic review. Stroke. (2001) 32:2433 RJ doi: 10.1161/hs1001.096009

19. Braun V, Clarke V. Using thematic analysis in psychology. Qualitative Research in Psychology. (2006) 3:77 Rese doi: 10.1191/1478088706qp063oa

20. Mputu PM, Beaus478088706qp063rd-Denis A. Mac-Thiong J-M. early predictors of neurological outcomes after traumatic spinal cord injury: a systematic review and proposal of a conceptual framework. Am J Phys Med Rehabil. (2021) 100:700Reha doi: 10.1097/PHM.0000000000001701

21. Benito J, DePuy V, Hardie E, Zamprognoa H, Thomson Simpson AW, et al. Reliability and discriminatory testing of a client-based metrology instrument, feline musculoskeletal pain index (FMPI) for the evaluation of degenerative joint disease-associated pain in cats. Veterinary J. (2013) 3:368ity doi: 10.1016/j.tvjl.2012.12.015

22. Benito J, Hansen B, DePuy V, Davidson GS, Thomson Simpson AW, et al. Feline musculoskeletal pain index: responsiveness and testing of criterion validity. J Veterinary Int Med. (2013) 3:474y In doi: 10.1111/jvim.12077

23. Gruen ME, Alfaro-Co rdoba M, Thomson AE, Worth AC, Staicu A-M. Lascelles BD. The use of functional data analysis to evaluate activity in a spontaneous model of degenerative joint disease associated pain in cats. PLoS ONE. (2017) 1:e0169576. doi: 10.1371/journal.pone.0169576
24. Lascelles DB, Hansen SR, DePuy V, Thomson A, Pierce CC, Smith ES, et al Evaluation of client-specific outcome measures and activity monitoring to measure pain relief in cats with osteoarthritis. J Vet Intern Med. (2007) 21:410ed. doi: 10.1111/j.1939-1676.2007.tb02983.x

25. Zamprogno H, Hansen BD, Bondell HD, Sumrell AT, Simpson W, Robertson ID, et al. Item generation and design testing of a questionnaire to assess degenerative joint diseasecats with osteoarthritis. AJVR. (2010) 12:1417nsen doi: 10.2460/ajvr.71.12.1417

26. Clarke SP. Bennett D. Feline osteoarthritis: a prospective study of 28 cases. J Small Animal Practice. (2006) 47:4391 Pr. doi: 10.1111/j.1748-5827.2006.00143.x

27. Morela I. Mills DS. Behavioural signs of pain in cats: an expert consensus. PLoS ONE. (2016) 2:e0150040. doi: 10.1371/journal.pone.0150040

28. Klinck MP, Frank D, Guillot M. Troncy E. Owner-perceived signs and veterinary diagnosis in 50 cases of feline osteoarthritis. CVJ. (2012) 53: $1181 n 6$.

29. Klinck MP, Gruen ME. del Castillo JR, Guillot M, Thomson AE, Heit M, et al. Development and preliminary validity and reliability of the Montr $\mathrm{M}$, et al.ry diagnosis in 50 cases of feline osteoarthritis.ionnaire to assess degenerative joint diseasecats with Appl. Anim. Behav. Sci. (2018) 200:96hav. doi: 10.1016/j.applanim.2017.11.013

30. Kranenburg HC, Meij BP, van Hofwegen EM, Voorhout G, Slingerland L, Picavet IP, et al. Prevalence of spondylosis deformans in the feline spine and correlation with owner-perceived behavioural changes. Vet Comp Orthop Traumato. (2012) 3:217hop doi: 10.3415/VCOT-11-06-0092

31. Roberts C, Armson B, Bartram D, Belshaw Z, Capon H, Cherry G, et al. Construction of a conceptual framework for assessment of health related quality of life in dogs with osteoarthritis. Frontiers. In Press.

32. Fatoye F, Fatoye S, Macmillan F, Rowe P, van der Linden M. Pain intensity and quality of life perception in children with hypermobility syndrome. Rheumatol Int. (2012) 32:1277. 4. doi: 10.1007/s00296-010-1729-2

Conflict of Interest: AW, KM, and IO work for Zoetis that funded the developed the conceptual framework.

The remaining authors declare that the research was conducted in the absence of any commercial or financial relationships that could be construed as a potential conflict of interest.

Publisher's Note: All claims expressed in this article are solely those of the authors and do not necessarily represent those of their affiliated organizations, or those of the publisher, the editors and the reviewers. Any product that may be evaluated in this article, or claim that may be made by its manufacturer, is not guaranteed or endorsed by the publisher.

Copyright (c) 2021 Yeowell, Burns, Fatoye, Gebrye, Wright, Mwacalimba and Odeyemi. This is an open-access article distributed under the terms of the Creative Commons Attribution License (CC BY). The use, distribution or reproduction in other forums is permitted, provided the original author(s) and the copyright owner(s) are credited and that the original publication in this journal is cited, in accordance with accepted academic practice. No use, distribution or reproduction is permitted which does not comply with these terms. 